\title{
SPACE-TIME REGULARITY OF SOLUTIONS OF THE PARABOLIC STOCHASTIC CAUCHY PROBLEM
}

\author{
JOHANNA DETTWEILER, JAN VAN NEERVEN, AND LUTZ WEIS
}

\begin{abstract}
We study space-time Hölder regularity of the solutions of the linear stochastic Cauchy problem

$$
\left\{\begin{aligned}
d U(t) & =A U(t) d t+d W(t), \quad t \in[0, T], \\
U(0) & =0
\end{aligned}\right.
$$

where $A$ is the generator of an analytic semigroup on a Banach space $E$ and $W$ is an $E$-valued Brownian motion. When $-A$ admits a $\gamma$-bounded $H^{\infty}$ calculus the solution is shown to have maximal regularity in the sense that $U$ has a modification with paths in $L^{2}\left(0, T ; \mathscr{D}\left((-A)^{\frac{1}{2}}\right)\right)$. The results are applied to prove optimal and maximal Hölder space-time regularity for second order parabolic stochastic partial differential equations.
\end{abstract}

\section{INTRODUCTION}

In this paper we study space-time regularity of the solutions of the linear stochastic Cauchy problem

$$
\left\{\begin{aligned}
d U(t) & =A U(t) d t+d W(t), \quad t \in[0, T], \\
U(0) & =0
\end{aligned}\right.
$$

where $A$ is the generator of an analytic $C_{0}$-semigroup $S=\{S(t)\}_{t \geqslant 0}$ on a real Banach space $E$ and $W$ is an $E$-valued Brownian motion. Assuming for simplicity that $S$ has negative growth bound, for Hilbert spaces $E$ it is well known that (1.1) admits a unique solution $U=\{U(t)\}_{t \in[0, T]}$ and that this solution has a modification with paths belonging to $C^{\eta}\left([0, T] ; \mathscr{D}\left((-A)^{\theta}\right)\right)$ for all $\eta, \theta \geqslant 0$ satisfying $\eta+\theta<\frac{1}{2}$. If the semigroup generated by $A$ is also contractive, then each $U(t)$ takes values in $\mathscr{D}\left((-A)^{\frac{1}{2}}\right)$ almost surely and the paths of $(-A)^{\frac{1}{2}} U$ belong to $\left.L^{2}(0, T ; E)\right)$ almost surely. An exposition of these results is presented in [9, Chapter 5], where further references may be found.

The first obstruction one meets in the Banach space setting is that for generators of general $C_{0}$-semigroups, the linear stochastic Cauchy problem (1.1) may fail to have a solution even if $W$ is a rank one Brownian motion. Examples with nonexistence are known for linear stochastic evolution equations in spaces $L^{p}(\mu)$ for $p \in[1,2)[23]$ and $C(K)[12]$. For generators of analytic $C_{0}$-semigroups this pathology does not occur and a unique solution $U$ of (1.1) always exists. The objective of this paper is to study the regularity properties of this solution. Our first main result (Theorem 4.1) describes the combined space-time regularity of $U$ with optimal

Date: June 19, 2006.

2000 Mathematics Subject Classification. Primary: 35R15; Secondary: 47D06, 60H15.

Key words and phrases. Parabolic stochastic evolution equations, analytic semigroups, spacetime Hölder regularity, $H^{\infty}$-calculus, maximal regularity, $R$-boundedness.

The second named author gratefully acknowledges support by a 'VIDI subsidie' in the 'Vernieuwingsimpuls' programme of the Netherlands Organization for Scientific Research (NWO) and by the Research Training Network HPRN-CT-2002-00281. The third named author was supported by grants from the Volkswagenstiftung (I/78593) and the Deutsche Forschungsgemeinschaft (We $2847 / 1-1)$. 
Hölder exponents. As an application we prove that under suitable assumptions on the coefficients, stochastic partial differential equations governed by second order uniformly elliptic operators on a bounded interval in space dimension one, driven by a space-time white noise, admit solutions which are simultaneously Hölder continuous in time of exponent $\alpha$ and Hölder continuous in space of exponent $\beta$, provided that $0 \leqslant 2 \alpha+\beta<\frac{1}{2}$.

Under the additional assumptions that $-A$ admits a $\gamma$-bounded $H^{\infty}$-calculus and the underlying Banach space $E$ has finite cotype, our second main result (Theorem 6.2 ) asserts that $U$ has maximal regularity, in the sense that $(-A)^{\frac{1}{2}} U$ has a modification with paths belonging to $L^{2}(0, T ; E)$. This is a natural extension of the corresponding maximal regularity result for analytic Hilbert space contraction semigroups mentioned earlier, since by the Sz.-Nagy dilation theorem and a recent result of Le Merdy [19] (see also [18, Section 11]) we know that if $A$ is the generator of an analytic semigroup on a Hilbert space $E$, then $-A$ admits a $\gamma$-bounded $H^{\infty}$-calculus if and only if $A$ generates an analytic contraction semigroup in some equivalent Hilbert norm on $E$. The result is applied to prove maximal regularity for a certain second order parabolic stochastic partial differential equation on bounded domains in $\mathbb{R}^{d}$.

Our examples show how our methods allow one to obtain sharp regularity results for stochastic partial differential equations by solving them directly in suitable Banach spaces.

\section{Preliminaries}

Throughout this paper, $H$ is a separable real Hilbert space, $E$ is a real Banach space, and $(\Omega, \mathscr{F}, \mathbb{P})$ is a probability space.

2.1. $\gamma$-Radonifying operators. Let $\left(\gamma_{n}\right)_{n \geqslant 1}$ be a Gaussian sequence on $(\Omega, \mathscr{F}, \mathbb{P})$. A bounded operator from a separable real Hilbert space $H$ with orthonormal basis $\left(h_{n}\right)_{n \geqslant 1}$ is said to be $\gamma$-radonifying if the sum $\sum_{n \geqslant 1} \gamma_{n} T h_{n}$ converges in $L^{2}(\Omega ; E)$. The space $\gamma(H, E)$ of all $\gamma$-radonifying operators from $H$ into $E$ is a Banach space with respect to the norm $\|\cdot\|_{\gamma(H, E)}$ defined by

$$
\|T\|_{\gamma(H, E)}^{2}:=\mathbb{E}\left\|\sum_{n \geqslant 1} \gamma_{n} T h_{n}\right\|^{2} .
$$

The space $\gamma(H, E)$ and its norm are independent of the choice of the basis $\left(h_{n}\right)_{n \geqslant 1}$.

The operator $T$ is said to be almost summing if the partial sums $\sum_{n=1}^{N} \gamma_{n} T h_{n}$ are uniformly bounded in $L^{2}(\Omega ; E)$. Every $\gamma$-radonifying operator is almost summing and we have

$$
\|T\|_{\gamma(H, E)}^{2}=\sup _{N \geqslant 1} \mathbb{E}\left\|\sum_{n=1}^{N} \gamma_{n} T h_{n}\right\|^{2}
$$

If $E$ does not contain a closed subspace isomorphic to $c_{0}$, then a celebrated theorem of Hoffmann-Jorgensen and Kwapień [20, Theorem 9.29] implies that every almost summing operator from $H$ to $E$ is $\gamma$-radonifying. For more information we refer to $[2,20,25,29]$.

Let $\mathscr{L}(H, E)$ denote the space of all bounded linear operators from $H$ to $E$. We say that a function $\Phi:(0, T) \rightarrow \mathscr{L}(H, E)$ belongs to $L^{2}(0, T ; H)$ scalarly if $t \mapsto \Phi^{*}(t) x^{*}$ belongs to $L^{2}(0, T ; H)$ for all $x^{*} \in E^{*}$. We say that such a function $\Phi$ represents an operator $T \in \mathscr{L}\left(L^{2}(0, T ; H), E\right)$ if

$$
\left\langle T f, x^{*}\right\rangle=\int_{0}^{T}\left[\Phi^{*}(t) x^{*}, f(t)\right]_{H} d t, \quad f \in L^{2}(0, T ; H), x^{*} \in E^{*} .
$$


Noting that $T$ is uniquely determined by $\Phi$ we sometimes write $T=I_{\Phi}$. This operator is adjoint to the operator $x^{*} \mapsto \Phi^{*}(\cdot) x^{*}$ from $E^{*}$ into $L^{2}(0, T ; H)$. We denote by $\gamma(0, T ; H, E)$ the vector space of all functions $\Phi:(0, T) \rightarrow \mathscr{L}(H, E)$ which represent a $\gamma$-radonifying operator $I_{\Phi} \in \mathscr{L}\left(L^{2}(0, T ; H), E\right)$, identifying functions representing the same operator. For a function $\Phi \in \gamma(0, T ; H, E)$ we define

$$
\|\Phi\|_{\gamma(0, T ; H, E)}:=\left\|I_{\Phi}\right\|_{\gamma\left(L^{2}(0, T ; H), E\right)} .
$$

We identify functions reprensting the same operator. It is easy to see that for all $\Phi \in \gamma(0, T ; H, E)$ the reflected function $t \mapsto \Phi(T-t)$ belongs to $\gamma(0, T ; H, E)$ with equal norm. Moreover, for all $t \in(0, T)$ the restriction $\left.\Phi\right|_{(0, t)}$ belongs to $\gamma(0, t ; H, E)$, and an easy application of the contraction principle gives

$$
\left\|\left.\Phi\right|_{(0, t)}\right\|_{\gamma(0, t ; H, E)} \leqslant\|\Phi\|_{\gamma(0, T ; H, E)} .
$$

The following simple lemma will be useful.

Lemma 2.1. If $g \in L^{2}(0, T)$ and $B \in \gamma(H, E)$, then the function $g B: t \mapsto g(t) B$ belongs to $\gamma(0, T ; H, E)$ and we have

$$
\|g B\|_{\gamma(0, T ; H, E)}=\|g\|_{L^{2}(0, T)}\|B\|_{\gamma(H, E)} .
$$

Proof. Let $\left(f_{m}\right)_{m \geqslant 1}$ and $\left(h_{n}\right)_{n \geqslant 1}$ be orthonormal bases for $L^{2}(0, T)$ and $H$, respectively, and note that $\left(f_{m} \otimes h_{n}\right)_{m, n \geqslant 1}$ is an orthonormal basis for $L^{2}(0, T ; H)$. Let $\left(\gamma_{m n}\right)_{m, n \geqslant 1}$ be a doubly indexed Gaussian sequence and define

$$
\xi_{n}:=\sum_{m \geqslant 1} \gamma_{m n} \int_{0}^{T} f_{m}(t) g(t) d t
$$

The sum defining each $\xi_{n}$ converges in $L^{2}(\Omega)$ and is $N\left(0,\|g\|_{2}^{2}\right)$-distributed, and the resulting i.i.d. sequence $\left(\xi_{n}\right)_{n \geqslant 1}$ is Gaussian.

Define $S: L^{2}(0, T ; H) \rightarrow E$ by

$$
S f:=\int_{0}^{T} g(t) B f(t) d t, \quad f \in L^{2}(0, T ; H) .
$$

Then $g B$ represents $S$ and we have

$$
\begin{aligned}
\|S\|_{\gamma\left(L^{2}(0, T ; H), E\right)}^{2} & =\mathbb{E}\left\|\sum_{m, n \geqslant 1} \gamma_{m n} \int_{0}^{T} f_{m}(t) g(t) B h_{n} d t\right\|^{2} \\
& =\mathbb{E}\left\|\sum_{n \geqslant 1} \xi_{n} B h_{n}\right\|^{2}=\|g\|_{2}^{2}\|B\|_{\gamma(H, E)}^{2} .
\end{aligned}
$$

For $H=\mathbb{R}$ the above definitions simplify by canonically identifying $\mathscr{L}(\mathbb{R}, E)$ with $E$. Accordingly, we say that a function $\phi:(0, T) \rightarrow E$ belongs to $L^{2}(0, T)$ scalarly if the scalar-valued function $\left\langle\phi, x^{*}\right\rangle$ belongs to $L^{2}(0, T)$ for all $x^{*} \in E^{*}$. Such a function is said to represent an operator $T \in \mathscr{L}\left(L^{2}(0, T), E\right)$ if

$$
\left\langle T f, x^{*}\right\rangle=\int_{0}^{T}\left\langle\phi(t), x^{*}\right\rangle f(t) d t, \quad f \in L^{2}(0, T), x^{*} \in E^{*},
$$

and we write $\phi \in \gamma(0, T ; E)$ if the operator $T=I_{\phi}$ is $\gamma$-radonifying. As before we define $\|\phi\|_{\gamma(0, T ; E)}:=\left\|I_{\phi}\right\|_{\gamma\left(L^{2}(0, T), E\right)}$. 
2.2. $\gamma$-Boundedness. Let $\left(\gamma_{n}\right)_{n \geqslant 1}$ be a Gaussian sequence. A family of operators $\mathscr{T} \subseteq \mathscr{L}(E)$ is called $\gamma$-bounded if there exists a constant $C$ such that for all finite sequences $\left(T_{n}\right)_{n=1}^{N} \subseteq \mathscr{T}$ and $\left(x_{n}\right)_{n=1}^{N} \subseteq E$ we have

$$
\mathbb{E}\left\|\sum_{n=1}^{N} \gamma_{n} T_{n} x_{n}\right\|^{2} \leqslant C^{2} \mathbb{E}\left\|\sum_{n=1}^{N} \gamma_{n} x_{n}\right\|^{2} .
$$

The best possible constant $C$ in this inequality is called the $\gamma$-bound of $\mathscr{T}$, notation $\gamma(\mathscr{T})$. Replacing the Gaussian sequence $\left(\gamma_{n}\right)_{n \geqslant 1}$ by a Rademacher sequence $\left(r_{n}\right)_{n \geqslant 1}$ we obtain the notion of an $R$-bounded family. The $R$-bound of $\mathscr{T}$ is denoted by $R(\mathscr{T})$. The notion of $R$-boundedness has been studied recently by a number of authors in connection with the Maximal Regularity problem in Banach spaces; see $[6,11,18,31]$ and the references given there.

By a standard randomization argument, every $R$-bounded family is $\gamma$-bounded and we have $\gamma(\mathscr{T}) \leqslant R(\mathscr{T})$. Furthermore, every $\gamma$-bounded family is uniformly bounded. If $E$ has finite cotype (the definition will be recalled below) the notions of $R$-boundedness and $\gamma$-boundedness are equivalent [13]. This follows from the fact that in any Banach space we have

$$
\mathbb{E}\left\|\sum_{n=1}^{N} r_{n} x_{n}\right\|^{2} \leqslant \frac{1}{2} \pi \mathbb{E}\left\|\sum_{n=1}^{N} \gamma_{n} x_{n}\right\|^{2} .
$$

while for Banach spaces with finite cotype there exists a constant $C$, depending only on $E$, such that

$$
\mathbb{E}\left\|\sum_{n=1}^{N} \gamma_{n} x_{n}\right\|^{2} \leqslant C^{2} \mathbb{E}\left\|\sum_{n=1}^{N} r_{n} x_{n}\right\|^{2} .
$$

We refer to $[13,20]$ for more details. In Hilbert spaces the notions of $\gamma$-boundedness and $R$-boundedness coincide with the notion of uniform boundedness.

We recall the following criterion for $R$-boundedness [31, Proposition 2.5]:

Lemma 2.2. If $\Psi:(0, T) \rightarrow \mathscr{L}(E)$ is continuously differentiable with integrable derivative, then the family $\mathscr{T}_{\Psi}:=\{\Psi(t): t \in(0, T)\}$ is $R$-bounded and

$$
R\left(\mathscr{T}_{\Psi}\right) \leqslant\|\Psi(T)\|+\int_{0}^{T}\left\|\Psi^{\prime}(t)\right\| d t
$$

The following multiplier result is a straightforward generalization of a result in [16], where it is formulated for the case $H=\mathbb{R}$. We call an operator-valued function $\Psi:(0, T) \rightarrow \mathscr{L}(E)$ strongly measurable if $\Psi x:(0, T) \rightarrow E, \Psi x(t):=\Psi(t) x$, is strongly measurable for all $x \in E$.

Lemma 2.3. If $\Psi:(0, T) \rightarrow \mathscr{L}(E)$ is strongly measurable and $\mathscr{T}_{\Psi}:=\{\Psi(t): t \in$ $(0, T)\}$ is $\gamma$-bounded, then for all $f \in \gamma(0, T ; H, E)$ the function $\Psi(\cdot) f(\cdot)$ belongs to $\gamma(0, T ; H, E)$ and

$$
\|\Psi(\cdot) f(\cdot)\|_{\gamma(0, T ; H, E)} \leqslant \gamma\left(\mathscr{T}_{\Psi}\right)\|f(\cdot)\|_{\gamma(0, T ; H, E)} .
$$

2.3. Stochastic integration. An $H$-cylindrical Brownian motion on a probability space $(\Omega, \mathscr{F}, \mathbb{P})$ is a family of bounded linear operators $W_{H}=\left\{W_{H}(t)\right\}_{t \geqslant 0}$ from $H$ into $L^{2}(\Omega)$ with the following properties:

(1) For all $h \in H,\left\{W_{H}(t) h\right\}_{t \geqslant 0}$ is a Brownian motion;

(2) For all $s, t \geqslant 0$ and $g, h \in H$ we have $\mathbb{E}\left(W_{H}(s) g \cdot W_{H}(t) h\right)=(s \wedge t)[g, h]_{H}$.

For a simple function of the form $\phi=\sum_{j=1}^{N} 1_{\left(t_{j-1}, t_{j}\right]} \otimes h_{j}$ with $0 \leqslant t_{0}<\cdots<$ $t_{N}<T$ and $h_{j} \in H, j=1, \ldots, N$, we define 


$$
\int_{0}^{T} \phi(t) d W_{H}(t):=\sum_{j=1}^{N} W_{H}\left(t_{j}\right) h_{j}-W_{H}\left(t_{j-1}\right) h_{j} .
$$

We have the Itô isometry

$$
\mathbb{E}\left\|\int_{0}^{T} \phi(t) d W_{H}(t)\right\|^{2}=\int_{0}^{T}\|\phi(t)\|_{H}^{2} d t
$$

which permits the extension of the stochastic integral to all of $L^{2}(0, T ; H)$. The following integration by parts formula, valid for $F \in C^{1}([0, T] ; H)$ is an easy generalization of its scalar counterpart:

$$
\int_{0}^{T} W_{H}(t) F^{\prime}(t) d t=W_{H}(T) F(T)-\int_{0}^{T} F(t) d W_{H}(t) .
$$

The definition of the stochastic integral for $H$-valued functions can be used, via duality, to define a stochastic integral for $\mathscr{L}(H, E)$-valued functions. Following [23], a function $\Phi:(0, T) \rightarrow \mathscr{L}(H, E)$ is said to be stochastically integrable with respect to $W_{H}$ if $\Phi$ belongs to $L^{2}(0, T ; H)$ scalarly and there exists an $E$-valued random variable $X_{\Phi}$ such that for all $x^{*} \in E^{*}$ we have

$$
\left\langle X_{\Phi}, x^{*}\right\rangle=\int_{0}^{T} \Phi^{*}(t) x^{*} d W_{H}(t)
$$

almost surely. The random variable $X_{\Phi}$, if it exists, is uniquely determined up to a null set and it is Gaussian. The following criterion for stochastic integrability, taken from [23], generalizes results from [9] (for Hilbert spaces E) and [4]:

Lemma 2.4. A function $\Phi:(0, T) \rightarrow \mathscr{L}(H, E)$ is stochastically integrable with respect to $W_{H}$ if and only if $\Phi \in \gamma(0, T ; H, E)$. In this situation we have

$$
\mathbb{E}\left\|\int_{0}^{T} \Phi(t) d W_{H}(t)\right\|^{2}=\|\Phi\|_{\gamma(0, T ; H, E)}^{2} .
$$

Noting that the stochastic integrability of $\Phi$ does not depend on the particular choice of $W_{H}$, we simply say that $\Phi$ is $H$-stochastically integrable.

\section{Existence And Uniqueness}

We shall be interested in the existence, uniqueness, and space-time regularity of solutions of the linear stochastic Cauchy problem

$$
\left\{\begin{aligned}
d U(t) & =A U(t) d t+B d W_{H}(t), \quad t \in[0, T], \\
U(0) & =0,
\end{aligned}\right.
$$

where $A$ is the generator of a $C_{0}$-semigroup $S=\{S(t)\}_{t \geqslant 0}$ on a real Banach space $E, B$ is a bounded operator from a separable real Hilbert space $H$ to $E$, and $W_{H}=\left\{W_{H}(t)\right\}_{t \geqslant 0}$ is an $H$-cylindrical Brownian motion on a probability space $(\Omega, \mathscr{F}, \mathbb{P})$. An $E$-valued process $U=\{U(t)\}_{t \in[0, T]}$ is called a weak solution of (3.1) if it is scalarly progressively measurable and for all $x^{*} \in \mathscr{D}\left(A^{*}\right)$, the domain of the adjoint operator $A^{*}$, the following two conditions are satisfied:

(1) Almost surely, the paths $t \mapsto U(t)$ are integrable on $(0, T)$;

(2) For all $t \in[0, T]$ we have, almost surely,

$$
\left\langle U(t), x^{*}\right\rangle=\int_{0}^{t}\left\langle U(s), A^{*} x^{*}\right\rangle d s+W_{H}(t) B^{*} x^{*}
$$

The following result was proved in [23] (see [4, 9] for special cases). 
Proposition 3.1. The problem (3.1) admits a weak solution $U=\{U(t)\}_{t \in[0, T]}$ if and only if $t \mapsto S(t) B$ is $H$-stochastically integrable on $(0, T)$. In this case, for all $0<t \leqslant T$ the function $s \mapsto S(t-s) B$ is $H$-stochastically integrable on $(0, t)$ and we have

$$
U(t)=\int_{0}^{t} S(t-s) B d W_{H}(s) \quad \text { almost surely. }
$$

In particular the solution $U$ is unique up to modification. Moreover,

$$
\mathbb{E}\|U(t)\|^{2}=\|S(t-\cdot) B\|_{\gamma(0, t ; H, E)}^{2}=\|S(\cdot) B\|_{\gamma(0, t ; H, E)}^{2} .
$$

Up to this point the operator $B$ was an arbitrary bounded operator from $H$ to $E$. From this point on we shall make the standing assumption that $B \in \gamma(H, E)$, i.e. the operator $B: H \rightarrow E$ is $\gamma$-radonifying. Under this assumption we may consider a fixed orthonormal basis $\left(h_{n}\right)_{n \geqslant 1}$ for $H$ and define

$$
W_{B}(t):=\sum_{n} W_{H}(t) h_{n} \otimes B h_{n}, \quad t \geqslant 0 .
$$

Since $B$ is $\gamma$-radonifying, for each $t \geqslant 0$ this series converges in $L^{2}(\Omega ; E)$. It is easy to check that the resulting process $W_{B}$ is an $E$-valued Brownian motion, which does not depend on the choice of the basis $\left(h_{n}\right)_{n \geqslant 1}$ up to indistinguishability. The problem (3.1) may be reformulated in terms of $W_{B}$ as follows:

$$
\left\{\begin{aligned}
d U(t) & =A U(t) d t+d W_{B}(t), \quad t \in[0, T], \\
U(0) & =0
\end{aligned}\right.
$$

Conversely, every $E$-valued Brownian motion $W$ can be represented in the form (3.2) by taking for $H$ the reproducing kernel Hilbert space of $W$ and for $B$ the canonical inclusion operator $H \hookrightarrow E$; see [4, 23] for more details.

We define an $E$-valued process $V=\{V(t)\}_{t \in[0, T]}$ by

$$
V(t):=\int_{0}^{t} S(t-s) W_{B}(s) d s, \quad t \in[0, T],
$$

where the integral on the right hand side is defined path by path and $V(0):=0$. Our first aim is to show that if the $C_{0}$-semigroup $S$ generated by $A$ is analytic, the process $W_{B}+A V$ is well defined and solves the Cauchy problem (3.1) on every interval $[0, T]$. For Hilbert spaces $E$ this result is well known, cf. [9, Chapter 5].

Proposition 3.2. Assume that $A$ generates an analytic $C_{0}$-semigroup on $E$ and let $B \in \gamma(H, E)$.

(i) The random variables $V(t)$ defined by (3.3) take values in $\mathscr{D}(A)$ almost surely and the process $\{A V(t)\}_{t \in[0, T]}$ has a continuous modification;

(ii) The $\mathscr{L}(H, E)$-valued function $S(\cdot) B$ is $H$-stochastically integrable on $(0, T)$, the problem (3.1) has a unique weak solution $U=\{U(t)\}_{t \in[0, T]}$, and for all $t \in[0, T]$ we have

$$
U(t)=W_{B}(t)+A V(t) \quad \text { almost surely. }
$$

Moreover this solution has a continuous modification.

Proof. Fix $0 \leqslant \beta<\frac{1}{2}$. For almost all $\omega \in \Omega, t \mapsto W_{B}(t, \omega)$ is $\beta$-Hölder continuous in $E$ and therefore by standard regularity results for the parabolic inhomogeneous Cauchy problem [22, Theorem 5.3.5], $t \mapsto V(t, \omega)=\int_{0}^{t} S(t-s) W_{B}(s, \omega) d s$ belongs to $C([0, T] ; \mathscr{D}(A))$. This proves $(1)$.

Let now $t \in[0, T]$ be fixed. To show that $t \mapsto \Phi(t):=S(t) B$ is $H$-stochastically integrable on $(0, t)$ and satisfies (3.4) we use the integration by parts identity (2.4). 
We apply it to the function $F \in C^{1}([0, t] ; H)$ given by $F(s)=\Phi^{*}(t-s) x^{*}$, where $x^{*} \in \mathscr{D}\left(A^{\odot}\right)$. Here, as usual, $A^{\odot}$ denotes the part of $A^{*}$ in $\overline{\mathscr{D}\left(A^{*}\right)}$. This gives

$$
-\left\langle A \int_{0}^{t} S(t-s) W_{B}(s) d s, x^{*}\right\rangle=\left\langle W_{B}(t), x^{*}\right\rangle-\int_{0}^{t} B^{*} S^{*}(t-s)^{*} x^{*} d W_{H}(t) .
$$

By the weak ${ }^{*}$-sequential denseness of $\mathscr{D}\left(A^{\odot}\right)$ in $E^{*}$, from [23, Theorem 2.3] we obtain that $S(t-\cdot) B$, and hence also $S(\cdot) B$, is $H$-stochastically integrable on $(0, t)$ and that (3.4) holds. This proves (2).

The main point of the proposition is the existence of a weak solution. Continuity of weak solutions for generators of analytic semigroups is proved in [4] without assumption $B \in \gamma(H, E)$; instead, the existence of a weak solution is explicitly assumed there.

Remark 3.3. In the case of a generator of an arbitrary $C_{0}$-semigroup, not necessarily analytic, the same method of proof shows that the process $V$ is well defined and continuous in extrapolation spaces of order $>\frac{1}{2}$. The identity (3.4) still holds, provided it is suitably interpreted in the extrapolation space.

\section{SpaCe-Time Regularity}

Having assured the existence of weak solutions, we proceed with investigating their regularity in space and time by carefully exploiting the smoothing effect of analytic semigroups. Our main result, Theorem 4.1, generalizes regularity results for the analytic case due to Da Prato and Zabczyk [9, Section 5.4] (for Hilbert spaces $E$ ) and Brzeźniak [3] (for martingale type 2 spaces $E$ ).

If $A$ is the generator of an analytic $C_{0}$-semigroup on $E$, then for real numbers $r>s(A)$ (the spectral bound of $A$ ), the fractional powers $(r-A)^{\theta}$ are well defined for all $\theta \in \mathbb{R}$. For $\theta>0$ we write $E_{\theta}:=\mathscr{D}\left((r-A)^{\theta}\right)$, which is a Banach space endowed with the norm $\|x\|_{E_{\theta}}=\left\|(r-A)^{\theta} x\right\|$. By well known results, as a set the space $E_{\theta}$ is independent of the choice of $r$, and its norm is equivalent to the usual graph norm.

Theorem 4.1. Assume that $A$ is the generator of an analytic $C_{0}$-semigroup $S$ on $E$, let $B \in \gamma(H, E)$, and let $U$ be the weak solution of problem (3.1). Let $\theta \geqslant 0$ and $\eta \geqslant 0$ satisfy $\theta+\eta<\frac{1}{2}$.

(1) The random variables $U(t)$ take values in $E_{\theta}$ almost surely and we have

$$
\mathbb{E}\|U(t)-U(s)\|_{E_{\theta}}^{2} \leqslant C|t-s|^{2 \eta}\|B\|_{\gamma(H, E)}^{2} \quad \forall t, s \in[0, T],
$$

with a constant $C$ independent of $B$;

(2) The process $U$ has a modification with paths in $C^{\eta}\left([0, T] ; E_{\theta}\right)$.

Remark 4.2. The theorem remains true if the fractional domain spaces $E_{\eta}$ are replaced by (real or complex) interpolation spaces and more generally, by spaces $E(\eta)$ satisfying inclusions $(E, \mathscr{D}(A))_{\eta, 1} \hookrightarrow E(\eta) \hookrightarrow(E, \mathscr{D}(A))_{\eta, \infty}$.

Before starting with the proof of the theorem we discuss the assumption that the operator $B: H \rightarrow E$ should be $\gamma$-radonifying. In certain interesting applications this assumption is not satisfied and even worse, the operator $B$ is unbounded. This situation arises for instance when a stochastic partial differential equation driven by white noise is formulated as an abstract stochastic evolution in a state space $E$. Typically, $E=E(\mathscr{O})$ will be a space of functions one some domain $\mathscr{O}$ in $\mathbb{R}^{d}$. The proper choice of $E(\mathscr{O})$ is suggested by the interpretation of the equation and the expected space regularity of its solutions. The natural choice for the Hilbert space $H$ used to model the white noise is then $L^{2}(\mathscr{O})$, with $B: L^{2}(\mathscr{O}) \rightarrow E(\mathscr{O})$ being the 
identity operator. However $L^{2}(\mathscr{O})$ may not embed into $E(\mathscr{O})$, and if it does, the embedding may fail to be $\gamma$-radonifying.

A way out of this difficulty is to interpret the equation in a suitably chosen Banach space $F$. Firstly, $E \cap F$ should be dense in both $E$ and $F$ and contain the range of $B$ (we think of $E$ and $F$ as being continuously embedded in some ambient locally convex topological vector space) and the part of $A$ in $E \cap F$ should extend uniquely to a generator $A_{F}$ of an analytic $C_{0}$-semigroup on $F$. Secondly, $B$ should extend to a $\gamma$-radonifying operator $B_{F}$ from $H$ into $F$. Now we can apply Theorem 4.1 in $F$ to the problem

$$
\left\{\begin{aligned}
d U(t) & =A_{F} U(t) d t+B_{F} d W_{H}(t) \quad t \in[0, T] \\
U(0) & =0
\end{aligned}\right.
$$

This solution will have its paths in $C^{\eta}\left([0, T] ; F_{\theta}\right)$ with $\eta, \theta \geqslant 0$ and $\eta+\theta<\frac{1}{2}$. If $F_{\theta}$ embeds continuously into $E$ the solutions take values in $E$ and are Hölder continuous in time of exponent $\eta$.

We will be primarily interested in the situation where we have a continuous dense embedding $E \hookrightarrow F$. At least in the case where $B$ is a bounded operator from $H$ to $E$, the above procedure gives a weak solution of the original problem in $E$ :

Proposition 4.3. Assume that $B: H \rightarrow E$ is bounded. Let $j: E \hookrightarrow F$ be a continuous and dense embedding, and assume that $A$ is the part in $E$ of an operator $A_{F}$ in $F$ which generates a $C_{0}$-semigroup on $F$. Suppose $U$ is an E-valued process whose trajectories belong to $L^{1}(0, T ; E)$ almost surely. If the $F$-valued process $j U$ is a weak solution of (4.1), then $U$ is a weak solution of (3.1).

Proof. It is clear that the defining properties of a weak solution are satisfied for functionals $x^{*} \in \mathscr{D}\left(A^{*}\right)$ of the form $x^{*}=j^{*} y^{*}$ with $y \in \mathscr{D}\left(A_{F}^{*}\right)$. As in the proof of [4, Theorem 5.3] (note that we may assume $E$ to be separable), from this one infers that these properties hold for all $x^{*} \in \mathscr{D}\left(A^{*}\right)$.

In the next section we will be interested in a version of this lemma for unbounded operators $B$. Assuming that $\mathscr{D}\left(A^{*}\right) \subseteq \mathscr{D}\left(B^{*}\right)$, the definition of a weak solution can be extended in a natural way. The resulting extension of problem (3.1) has been studied in [30, Appendix], where it is shown that at least for analytic generators $A$, Proposition 3.1 can be generalized if $(-A)^{\delta} B$ is bounded for some $0<\delta<\frac{1}{2}$ (this assumption is satisfied in the example in the next section). Proposition 4.3 extends to this setting as well.

We proceed with a simple illustration of the above ideas. A more elaborate example will be worked out in the next section.

Example 4.4. (Simultaneously diagonalizable case). Let $A$ be a diagonal operator on $E=l^{p}, 1 \leqslant p<\infty$, with real eigenvalues $-\lambda_{n}$ satisfying $\lambda_{n} \geqslant c$ for some $c>0$. Fix $\alpha \in(0,1)$ and define $F$ as the space of all real sequences $\left(x_{n}\right)$ such that $\left(\lambda_{n}^{-\alpha} x_{n}\right) \in l^{p}$. Endowed with the norm $\left\|\left(x_{n}\right)\right\|_{F}=\left\|\left(\lambda_{n}^{-\alpha} x_{n}\right)\right\|_{l^{p}}$, the space $F$ is a Banach space, and we have $E \hookrightarrow F$ with a continuous and dense embedding. Let $\left(b_{n}\right)$ be a sequence of nonnegative real numbers. The diagonal operator $B:\left(y_{n}\right) \mapsto$ $\left(b_{n} y_{n}\right)$ defines an element of $\gamma\left(l^{2}, F\right)$ if and only if $B_{-\alpha}:\left(y_{n}\right) \mapsto\left(\lambda_{n}^{-\alpha} b_{n} y_{n}\right)$ defines an element of $\gamma\left(l^{2}, l^{p}\right)$. By standard square function estimates the latter happens if and only if $\sum_{n} \lambda_{n}^{-\alpha p} b_{n}^{p}<\infty$. For the special case $b_{n}=1$ (the white noise case), it follows that $B$ defines an element of $\gamma\left(l^{2}, F\right)$ if and only $\left(\lambda_{n}^{-\alpha}\right) \in l^{p}$. Note that this condition depends on both $\alpha$ and $p$ and is likely to be fulfilled if $\alpha$ and/or $p$ are large enough. Also note that for $\theta>\alpha$ we have $F_{\theta} \hookrightarrow E=l^{p}$ with continuous inclusion. 
Proof of Theorem 4.1. We will use the notation ' $\lesssim$ for estimates involving constants which are independent of $B$.

Without loss of generality we assume that $\eta>0$. In order to bring out the idea of the proof we begin with a formal computation. Take $r$ large enough and put

$$
R(t):=A_{r}^{\theta} S(t), \quad t>0,
$$

where $A_{r}:=r-A$. Then,

$$
\begin{aligned}
(\mathbb{E} \| U & \left.(t+h)-U(t) \|_{E_{\theta}}^{2}\right)^{\frac{1}{2}} \\
= & \left(\mathbb{E}\left\|A_{r}^{\theta}[U(t+h)-U(t)]\right\|^{2}\right)^{\frac{1}{2}} \\
= & \left(\mathbb{E}\left\|\int_{0}^{t+h} R(t+h-s) B d W_{H}(s)-\int_{0}^{t} R(t-s) B d W_{H}(s)\right\|^{2}\right)^{\frac{1}{2}} \\
& \leqslant\left(\mathbb{E}\left\|\int_{t}^{t+h} R(t+h-s) B d W_{H}(s)\right\|^{2}\right)^{\frac{1}{2}} \\
& \quad+\left(\mathbb{E}\left\|\int_{0}^{t} R(t+h-s) B-R(t-s) B d W_{H}(s)\right\|^{2}\right)^{\frac{1}{2}} \\
= & \|R(\cdot) B\|_{\gamma(0, h ; H, E)}+\|R(\cdot+h) B-R(\cdot) B\|_{\gamma(0, t ; H, E)} \\
\leqslant & \|R(\cdot) B\|_{\gamma(0, h ; H, E)}+\|R(\cdot+h) B-R(\cdot) B\|_{\gamma(0, T ; H, E)} .
\end{aligned}
$$

where the final estimate follows from $(2.2)$.

If we can show that $R(\cdot) B \in \gamma(0, T ; H, E)$, then $R(\cdot) B$ is stochastically integrable with respect to $W_{H}$ by Lemma 2.4 and the above computation can be justified by noting that $A_{r}^{\theta}$ is an isomorphism from $E_{\theta}$ onto $E$. Assertion (i) will follow if we can show that for small $h$, say for $h \in(0,1)$, we have

$$
\|R(\cdot) B\|_{\gamma(0, h ; H, E)} \lesssim h^{\eta}\|B\|_{\gamma(H, E)}
$$

and

$$
\|R(\cdot+h) B-R(\cdot) B\|_{\gamma(0, T ; H, E)} \lesssim h^{\eta}\|B\|_{\gamma(H, E)} .
$$

We prove these estimates in two steps.

Step $1-$ Fix an arbitrary $\alpha \in\left[\theta+\eta, \frac{1}{2}\right)$ and $h \in(0,1)$. We first check that the two families

$$
\mathscr{T}_{h}:=\left\{s^{\alpha} R(s): s \in(0, h)\right\}
$$

and

$$
\mathscr{T}^{h}:=\left\{s^{\alpha}[R(s+h)-R(s)]: s \in(0, T)\right\}
$$

are $\gamma$-bounded, and that for small $h$ their $\gamma$-bounds satisfy

$$
\gamma\left(\mathscr{T}_{h}\right) \lesssim h^{\eta}
$$

and

$$
\gamma\left(\mathscr{T}^{h}\right) \lesssim h^{\eta}
$$

To prove (4.2) we apply Lemma 2.2 to the function $\Psi(s):=s^{\alpha} R(s)$ and check that its derivative

$$
\Psi^{\prime}(s)=s^{\alpha} A R(s)+\alpha s^{\alpha-1} R(s)
$$

is integrable on $(0, h)$. Using the analyticity of $S$ we have

$$
\|A R(s)\| \leqslant\left\|A_{r} R(s)\right\|+r\|R(s)\| \lesssim s^{-(1+\theta)}+s^{-\theta} \lesssim s^{-(1+\theta)}
$$

and we can estimate the first term in (4.4) by

$$
\int_{0}^{h} s^{\alpha}\|A R(s)\| d s \lesssim \int_{0}^{h} s^{\alpha-(1+\theta)} d s \leqslant \int_{0}^{h} s^{\eta-1} d s \lesssim h^{\eta},
$$


where we used that $\alpha-\theta \geqslant \eta$. Similarly, for the second term in (4.4) we have

$$
\int_{0}^{h} s^{\alpha-1}\|R(s)\| d s \lesssim \int_{0}^{h} s^{(\alpha-1)-\theta} d s \lesssim h^{\eta}
$$

Together with the estimate

$$
\left\|h^{\alpha} R(h)\right\| \lesssim h^{\alpha-\theta} \leqslant h^{\eta}
$$

we see that (4.2) follows from Lemma 2.2.

To prove (4.3) we apply Lemma 2.2 to the function $\Psi(s):=s^{\alpha}[R(s+h)-R(s)]$ and check that its derivative

$$
\Psi^{\prime}(s)=s^{\alpha} A[R(s+h)-R(s)]+\alpha s^{\alpha-1}[R(s+h)-R(s)]
$$

is integrable on $(0, T)$. For the first term in (4.5) we have

$$
\begin{aligned}
\int_{0}^{T} s^{\alpha}\|A[R(s+h)-R(s)]\| d s & =\int_{0}^{T} s^{\alpha}\left\|\int_{s}^{s+h} A^{2} R(u) d u\right\| d s \\
& \lesssim \int_{0}^{T} s^{\alpha}\left(\int_{s}^{s+h} u^{-2-\theta} d u\right) d s \\
& \lesssim h^{\alpha-\theta} \underbrace{\int_{0}^{\infty} \sigma^{\alpha}\left[(\sigma+1)^{-1-\theta}-\sigma^{-1-\theta}\right] d \sigma}_{<\infty} \lesssim h^{\eta} .
\end{aligned}
$$

Similarly, for the second term in (4.5) we have

$$
\begin{aligned}
\int_{0}^{T} s^{\alpha-1}\|R(s+h)-R(s)\| d s & \lesssim \int_{0}^{T} s^{\alpha-1}\left(\int_{s}^{s+h} u^{-1-\theta} d u\right) d s \\
& \lesssim h^{\alpha-\theta} \underbrace{\int_{0}^{\infty} \sigma^{\alpha-1}\left[(\sigma+1)^{-\theta}-\sigma^{-\theta}\right] d \sigma}_{<\infty} \lesssim h^{\eta} .
\end{aligned}
$$

Finally,

$$
\begin{aligned}
T^{\alpha}\|R(T+h)-R(T)\| & \lesssim T^{\alpha} \int_{T}^{T+h}\|A R(s)\| d s \\
& \lesssim T^{\alpha} \int_{T}^{T+h} s^{-1-\theta} d s \\
& \lesssim T^{\alpha}\left[(T+h)^{-\theta}-T^{-\theta}\right] \\
& \lesssim h^{\alpha-\theta} \underbrace{\left(\sup _{t \in \mathbb{R}_{+}} t^{\alpha}\left[(t+1)^{-\theta}-t^{-\theta}\right]\right)}_{<\infty} \lesssim h^{\eta} .
\end{aligned}
$$

Combination of these estimates gives (4.3).

Step 2-We combine Step 1 with Lemma 2.3. Recalling that $\alpha<\frac{1}{2}$, with Lemma 2.1 we obtain, with $\tau_{-\alpha}(t):=t^{-\alpha}$,

$$
\|R(\cdot) B\|_{\gamma(0, h ; H, E)} \lesssim h^{\eta}\left\|\tau_{-\alpha} B\right\|_{\gamma(0, h ; H, E)} \leqslant h^{\eta}\left\|\tau_{-\alpha}\right\|_{L^{2}(0, T)}\|B\|_{\gamma(H, E)}
$$

and

$$
\begin{aligned}
\| R(\cdot+h) & B-R(\cdot) B \|_{\gamma(0, T ; H, E)} \\
& \lesssim h^{\eta}\left\|\tau_{-\alpha} B\right\|_{\gamma(0, T ; H, E)} \leqslant h^{\eta}\left\|\tau_{-\alpha}\right\|_{L^{2}(0, T)}\|B\|_{\gamma(H, E)} .
\end{aligned}
$$

This concludes the proof of (i).

To prove (ii) we apply (i) with exponents $\eta^{\prime}$ and $\theta$, where $\eta^{\prime}>\eta$ is such that we still have $\eta^{\prime}+\theta<\frac{1}{2}$. By the Kahane-Khinchine inequalities we have, for any $q \geqslant 1$,

$$
\left(\mathbb{E}\|U(t)-U(s)\|_{E_{\theta}}^{q}\right)^{\frac{1}{q}} \lesssim\left(\mathbb{E}\|U(t)-U(s)\|_{E_{\theta}}^{2}\right)^{\frac{1}{2}} \lesssim|t-s|^{\eta^{\prime}}\|B\|_{\gamma(H, E)}
$$


The Kolmogorov-Chentsov continuity theorem now shows that $U$ has a modification $\tilde{U}$ which is Hölder continuous, for any exponent less than $\left(\eta^{\prime} q-1\right) / q$. Since $q$ can be chosen arbitrarily large, it follows that the paths of $\tilde{U}$ belong to $C^{\eta}\left([0, T] ; E_{\theta}\right)$ almost surely.

\section{An example}

We consider the following stochastic partial differential equation driven by spatiotemporal white noise:

$$
\left\{\begin{array}{rlrl}
\frac{\partial u}{\partial t}(t, x) & =L u(t, x)+\frac{\partial w}{\partial t}(t, x), & x \in[0,1], & t \in[0, T], \\
u(0, x) & =0, & x \in[0,1], \\
u(t, 0) & =u(t, 1)=0, & t \in[0, T],
\end{array}\right.
$$

where $L$ is a uniformly elliptic operator of the form

$$
L f(x)=a(x) f^{\prime \prime}(x)+b(x) f^{\prime}(x)+c(x) f(x), \quad x \in[0,1],
$$

with coefficients $a \in C^{\epsilon}[0,1]$ for some $\epsilon>0$ and $b, c \in L^{\infty}(0,1)$.

In what follows we let $H=L^{2}(0,1)$ and $E=L^{p}(0,1)$, where the exponent $p \geqslant 2$ is to be chosen later on. The realization of $L$ in $E$, henceforth denoted by $A$, satisfies the assumptions of Theorem $4.1[1,21]$. However, if we try to formulate the problem (5.1) as an abstract stochastic evolution equation in $E$ of the form

$$
\left\{\begin{aligned}
d U(t) & =A U(t) d t+I d W_{H}(t), \quad t \geqslant 0, \\
U(0) & =0
\end{aligned}\right.
$$

where $W_{H}$ is an $H$-cylindrical Brownian motion, we encounter the problem described in the previous section, namely that the identity operator $I$ is unbounded as an operator from $H$ into $E$. In order to overcome this problem we shall interpret the problem in a suitable extrapolation space of $E$.

We fix $\delta>\frac{1}{4}$ and let $E_{-\delta}$ denote the extrapolation space of order $\delta$ associated with $A$, i.e., $E_{-\delta}$ is the completion of $E$ with respect to the norm $\|x\|_{-\delta}:=$ $\left\|(-A)^{-\delta} x\right\|$. Since $A$ is invertible, $(-A)^{\delta}$ acts as an isomorphism from $E$ onto $E_{-\delta}$. We will show next that the identity operator $I$ on $H$ extends to a bounded embedding from $H$ into $E_{-\delta}$ which is $\gamma$-radonifying.

Let $\Delta_{H}$ and $A_{H}$ denote the realizations in $H$ of $\Delta$ and $A$ with Dirichlet boundary conditions, respectively. As is well known we have

$$
H_{1}:=\mathscr{D}\left(A_{H}\right)=H^{2,2} \cap H_{0}^{1,2}=\mathscr{D}\left(\Delta_{H}\right)=: H_{1}^{\Delta}
$$

with equivalent norms. Similarly,

$$
E_{1}:=\mathscr{D}(A)=H^{2, p} \cap H_{0}^{1, p}=\mathscr{D}(\Delta)=: E_{1}^{\Delta}
$$

with equivalent norms. By the results of [10], for $r>0$ large enough both $r-A$ and $-\Delta$ have bounded imaginary powers. Fixing such an $r$, by complex interpolation $[21,22]$ we obtain

$$
E_{1-\delta}^{\Delta}:=\mathscr{D}\left((-\Delta)^{1-\delta}\right)=\left(E, E_{1}^{\Delta}\right)_{1-\delta}=\left(E, E_{1}\right)_{1-\delta}=\mathscr{D}\left((r-A)^{1-\delta}\right)=: E_{1-\delta}
$$

with equivalent norms.

The functions $h_{n}(x):=\sqrt{2} \sin (n \pi x), n \geqslant 1$, form an orthonormal basis of eigenfunctions for $\Delta_{H}$ with eigenvalues $-\lambda_{n}$, where $\lambda_{n}=(n \pi)^{2}$. If we endow $H_{1}^{\Delta}$ with the equivalent Hilbert norm $\|f\|_{H_{1}^{\Delta}}:=\left\|\Delta_{H} f\right\|_{H}$, the functions $\lambda_{n}^{-1} h_{n}$ form an 
orthonormal basis for $H_{1}^{\Delta}$ and we have

$$
\begin{aligned}
\mathbb{E}\left\|\sum_{n \geqslant 1} \gamma_{n} \lambda_{n}^{-1} h_{n}\right\|_{E_{1-\delta}^{\Delta}}^{2} & =\mathbb{E}\left\|\sum_{n \geqslant 1} \gamma_{n} \lambda_{n}^{-1}(-\Delta)^{1-\delta} h_{n}\right\|_{E}^{2} \\
& =\mathbb{E}\left\|\sum_{n \geqslant 1} \gamma_{n}(n \pi)^{-2 \delta} h_{n}\right\|_{E}^{2} \stackrel{(*)}{\lesssim} \sum_{n \geqslant 1}(n \pi)^{-4 \delta},
\end{aligned}
$$

where $(*)$ follows from a standard square function estimate together with the fact that $\left\|h_{n}\right\|_{E} \leqslant \sqrt{2}$. The right hand side of (5.2) is finite since we took $\delta>\frac{1}{4}$.

It follows from (5.2) that the identity operator on $\mathscr{D}\left(\Delta_{H}\right)$ extends to a continuous embedding from $\mathscr{D}\left(\Delta_{H}\right)$ into $E_{1-\delta}^{\Delta}$ which is $\gamma$-radonifying; ; see also [3, Lemma 6.5]. Denoting by $E_{-\delta}$ the extrapolation space of order $\delta$ of $E$ associated with $A-r$, we obtain a commutative diagram

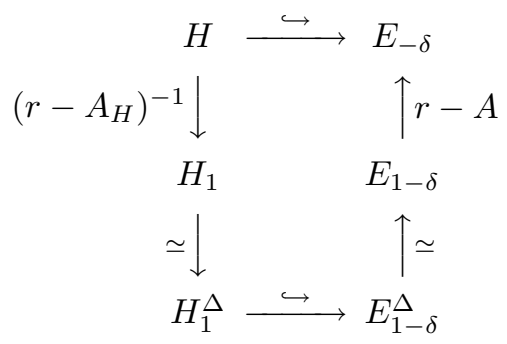

The inclusion $H_{1}^{\Delta} \hookrightarrow E_{1-\delta}^{\Delta}$ being $\gamma$-radonifying, the ideal property of $\gamma$-radonifying operators implies that the resulting embedding from $H$ into $E_{-\delta}^{\Delta}$ in the top line of the diagram is $\gamma$-radonifying; this operator is an extension of the identity operator on $H$. We shall denote this embedding by $I_{-\delta}$.

We are now in a position to apply Theorem 4.1. Fix arbitrary real numbers $\alpha, \beta, \theta$ satisfying $0 \leqslant 2 \alpha+\beta<\frac{1}{2}, \frac{1}{4}<\delta<\theta, \alpha+\theta<\frac{1}{2}$, and $\beta<2 \theta-2 \delta$. Put $\eta:=\theta-\delta$. Since the extrapolated operator $A_{-\delta}$ generates an analytic $C_{0}$-semigroup in $E_{-\delta}$ we may apply Theorem 4.1 in the space $E_{-\delta}$ to obtain a weak solution $U$ of the problem

$$
\left\{\begin{aligned}
d U(t) & =A_{-\delta} U(t) d t+I_{-\delta} d W_{H}(t), \quad t \in[0, T], \\
U(0) & =0
\end{aligned}\right.
$$

with paths in the space $C^{\alpha}\left([0, T] ;\left(E_{-\delta}\right)_{\theta}\right)=C^{\alpha}\left([0, T] ; E_{\eta}\right)$. Noting that $\beta<2 \eta$ we choose $p$ so large that $\beta+\frac{1}{p}<2 \eta$. We have

$$
E_{\eta}=E_{\eta}^{\Delta}=H_{0}^{2 \eta, p}=\left\{f \in H^{2 \eta, p}: f(0)=f(1)=0\right\}
$$

with equivalent norms [28]. By the Sobolev embedding theorem,

$$
H^{2 \eta, p} \hookrightarrow c^{\beta}[0,1]
$$

with continuous inclusion. Here $c^{\beta}[0,1]$ is the space of all continuous functions $f:[0,1] \rightarrow \mathbb{R}$ for which

$$
\lim _{\delta \downarrow 0} \sup _{|t-s| \leqslant \delta} \frac{|f(t)-f(s)|}{|t-s|^{\beta}}=0 .
$$

Endowed with the norm

$$
\|f\|_{c^{\beta}[0,1]}:=\|f\|+\sup _{t \neq s} \frac{|f(t)-f(s)|}{|t-s|^{\beta}}
$$

this space is a separable Banach space. We denote $c_{0}^{\beta}[0,1]=\left\{f \in c^{\beta}[0,1]: f(0)=\right.$ $f(1)=0\}$. Putting things together we obtain a continuous inclusion

$$
E_{\eta} \hookrightarrow c_{0}^{\beta}[0,1]
$$


In particular it follows that $U$ takes values in $E$. Almost surely, the trajectories of $U$ belong to $C^{\alpha}\left([0, T] ; c_{0}^{\beta}[0,1]\right)$. In particular, the trajectories of $U$ belong to $L^{1}(0, T ; E)$ almost surely. In view of Proposition 4.3 and the discussion following it, we have proved the following theorem.

Theorem 5.1. Let $\alpha$ and $\beta$ be real numbers satisfying $0 \leqslant 2 \alpha+\beta<\frac{1}{2}$. Under the above assumptions on $L$, the problem (5.1) admits a weak solution in $L^{p}(0,1)$ for all $1 \leqslant p<\infty$, and this solution has paths in $C^{\alpha}\left([0, T] ; c_{0}^{\beta}[0,1]\right)$.

Note that the ranges of the admissible Hölder exponents in the theorem are independent of the operator $L$.

For $L=\Delta$ the existence of a solution in $C^{\alpha}([0, T] \times[0,1])$ for $0 \leqslant \alpha<\frac{1}{4}$ was proved by Da Prato and Zabczyk by very different methods, see [8] and [9, Theorem 5.20]. This result was improved by Brzeźniak [18], who obtained Theorem 5.1 for $L=\Delta$ and noted without proof the possible extension to a more general class of second order elliptic operators.

An extension of Theorem 5.1 to operators of order $2 m$ on domains in higher dimensions will be presented elsewhere.

Related equations have been studied by many authors and with different methods; see for example [5, 9] and the references given there.

\section{Maximal Regularity}

In this section we will sharpen Theorem 4.1 in the case where $-A$ admits a $\gamma$ bounded $H^{\infty}$-calculus. Under this assumption we will prove maximal regularity of the weak solution. Our approach requires finite cotype of the underlying Banach space.

Recall that a Banach space $E$ is said to be of cotype $q$, where $q \in[2, \infty)$, if there is a constant $C$ such that for all finite sequences $\left(x_{n}\right)_{n=1}^{N}$ in $E$ we have

$$
\left(\sum_{n=1}^{N}\left\|x_{n}\right\|^{q}\right)^{1 / q} \leqslant C\left(\mathbb{E}\left\|\sum_{n=1}^{N} r_{n} x_{n}\right\|^{2}\right)^{\frac{1}{2}},
$$

where $\left(r_{n}\right)_{n=1}^{N}$ is a sequence of independent Rademacher variables. The Banach space $E$ is said to have finite cotype if it has cotype $q$ for some $q \in[2, \infty)$.

For $0<\omega<\pi$ we let $\Sigma_{\omega}:=\{\lambda \in \mathbb{C}: \lambda \neq 0,|\arg \lambda|<\omega\}$. A densely defined operator $-A$ is called sectorial if $A$ is injective, has dense range, and for some $0<\omega<\pi$ we have $\sigma(-A) \subseteq \overline{\Sigma_{\omega}}$ and

$$
\|\lambda R(\lambda,-A)\| \leqslant C_{\omega} \quad \forall \lambda \in \complement \overline{\Sigma_{\omega}}
$$

with constant $C_{\omega}$ independent of $\lambda$. The infimum of all such $\omega$ is denoted by $\omega(-A)$. Recall that if $-A$ is sectorial with $0<\omega(-A)<\frac{\pi}{2}$ if and only if $A$ generates an analytic $C_{0}$-semigroup $(S(t))_{t \geqslant 0}$ which is uniformly bounded on some sector containing the interval $(0, \infty)[14,18]$.

For $0<\omega<\pi$ we write $H_{0}^{\infty}\left(\Sigma_{\omega}\right)=\bigcup_{\varepsilon>0} H_{\varepsilon}^{\infty}\left(\Sigma_{\omega}\right)$, where $H_{\varepsilon}^{\infty}\left(\Sigma_{\omega}\right)$ is the class of bounded analytic functions $f: \Sigma_{\omega} \rightarrow \mathbb{C}$ which satisfy an estimate

$$
|f(\lambda)| \leqslant\left(\frac{|\lambda|}{1+|\lambda|^{2}}\right)^{\varepsilon}, \quad \lambda \in \Sigma_{\omega} .
$$

If $-A$ is sectorial with $0<\omega(-A)<\pi$, then for all $\omega(-A)<\sigma<\omega<\pi$ and $f \in H_{0}^{\infty}\left(\Sigma_{\omega}\right)$ we can define a bounded operator $f(-A)$ by the Dunford formula

$$
f(-A)=\frac{1}{2 \pi i} \int_{\partial \Sigma_{\sigma}} f(\lambda) R(\lambda,-A) d \lambda .
$$


If we have an estimate

$$
\|f(-A)\| \leqslant C_{\omega}\|f\|_{H^{\infty}\left(\Sigma_{\omega}\right)}, \quad f \in H_{0}^{\infty}\left(\Sigma_{\omega}\right),
$$

where $\|\cdot\|_{H^{\infty}\left(\Sigma_{\omega}\right)}$ denotes the supremum norm on $\Sigma_{\omega}$ and the constant $C_{\omega}$ is independent of $f$, it is possible to extend the definition of $f(-A)$ to all functions $f \in H^{\infty}\left(\Sigma_{\omega}\right)$ and we say that $-A$ admits a bounded $H^{\infty}\left(\Sigma_{\omega}\right)$-calculus. We say that $-A$ admits a bounded $H^{\infty}$-calculus if it admits a bounded $H^{\infty}\left(\Sigma_{\omega}\right)$-calculus for some $0<\omega<\pi$. The infimum of all such $\omega$ is denoted by $\omega_{\infty}(-A)$. The notion of a bounded $H^{\infty}$-calculus was introduced McIntosh and his collaborators and has been studied since then by many authors; we refer to [7].

If $f$ admits a bounded $H^{\infty}\left(\Sigma_{\omega}\right)$ calculus and the set

$$
\left\{f(-A):\|f\|_{H^{\infty}\left(\Sigma_{\omega}\right)} \leqslant 1\right\}
$$

is $\gamma$-bounded, we say that $-A$ has a $\gamma$-bounded $H^{\infty}\left(\Sigma_{\omega}\right)$-calculus. We say that $-A$ admits a $\gamma$-bounded $H^{\infty}$-calculus if it admits a $\gamma$-bounded $H^{\infty}\left(\Sigma_{\omega}\right)$-calculus for some $0<\omega<\pi$, and the number $\omega_{\infty}^{\gamma}(-A)$ is defined as before. For more details we refer to $[11,15,16,18]$.

If $E$ has Pisier's property $(\alpha)[26]$, then $-A$ admits a bounded $H^{\infty}$-calculus if and only if $-A$ admits a $\gamma$-bounded $H^{\infty}$-calculus and one has $\omega_{\infty}(-A)=\omega_{\infty}^{\gamma}(-A)$. Examples of spaces with property $(\alpha)$ are all Hilbert spaces, $L^{p}$-spaces for $1 \leqslant p<$ $\infty$, and spaces isomorphic to closed subspaces of these.

On a Hilbert space $E$, negative generators of $C_{0}$-contraction semigroups, as well as negative generators given by closed sectorial forms, admit a $\gamma$-bounded $H^{\infty}$ calculus. It is also known that a large class of elliptic partial differential operators on regular bounded domains in $\mathbb{R}^{d}$ admit a $\gamma$-bounded $H^{\infty}$-calculus (see $[11,18]$ ).

The following lemma is well known and is stated for the convenience of the reader. See [10, Lemma 3.1] for a related result. We use the notation $B_{l \infty}$ for the closed unit ball of $l^{\infty}$.

Lemma 6.1. Assume that $-A$ admits a $\gamma$-bounded $H^{\infty}$-calculus of angle $0<$ $\omega_{\infty}(-A)<\pi$. Fix a function $f \in H_{0}^{\infty}\left(\Sigma_{\sigma}\right)$, where $\omega<\sigma<\pi$. Then the family

$$
F=\left\{\sum_{n=1}^{N} a_{n} f\left(-2^{-n} s A\right): N \geqslant 1, s>0, a \in B_{l \infty}\right\}
$$

is $\gamma$-bounded, with $\gamma$-bound depending only on $A$ and $\sigma$.

Proof. For $N \geqslant 1, s>0$, and $a \in B_{l \infty}$ fixed, define $f_{N, s, a}: \Sigma_{\sigma} \rightarrow \mathbb{C}$ by

$$
f_{N, s, a}(\lambda):=\sum_{n=1}^{N} a_{n} f\left(2^{-n} s \lambda\right) .
$$

Since $f \in H_{\varepsilon}^{\infty}\left(\Sigma_{\sigma}\right)$ for some $\varepsilon>0$,

$$
\left|f_{N, s, a}(\lambda)\right| \leqslant \sum_{n=1}^{N}\left(\frac{2^{-n} s|\lambda|}{1+\left(2^{-n} s|\lambda|\right)^{2}}\right)^{\varepsilon}=: M(s|\lambda|) .
$$

It is elementary to check that $\sup _{r>0} M(r)<\infty$, and therefore the family $\left\{f_{N, a, j}\right.$ : $\left.N \geqslant 1, s>0, a \in B_{l}\right)$ is uniformly bounded in $H^{\infty}\left(\Sigma_{\sigma}\right)$. The result now follows from the fact that $-A$ admits a $\gamma$-bounded $H^{\infty}\left(\Sigma_{\sigma}\right)$-calculus.

The main result of this section, which generalizes e.g. [9, Proposition A.19], reads as follows.

Theorem 6.2. Let $E$ have finite cotype and assume that $-A$ admits $\gamma$-bounded $H^{\infty}$-calculus of angle $0<\omega_{\infty}^{\gamma}(-A)<\frac{\pi}{2}$. Then the solution $U$ of problem (3.1) has 
maximal regularity in the sense that for all $t \in[0, T]$ we have $U(t) \in \mathscr{D}\left((-A)^{\frac{1}{2}}\right)$ almost surely and

$$
\mathbb{E}\left\|(-A)^{\frac{1}{2}} U(t)\right\|^{2} \leqslant C\|B\|_{\gamma(H, E)}^{2}
$$

for a suitable constant $C$ independent of $T>0, t \in[0, T]$, and $B \in \gamma(H, E)$. Moreover, $(-A)^{\frac{1}{2}} U$ is continuous in all moments, i.e., for all $1 \leqslant p<\infty$ we have

$$
\lim _{s \rightarrow t} \mathbb{E}\left\|(-A)^{\frac{1}{2}}(U(t)-U(s))\right\|^{p}=0,
$$

Finally, the paths of $(-A)^{\frac{1}{2}} U$ belong to $L^{2}(0, T ; E)$ almost surely.

Proof. Following [16] we consider the function $\psi(\lambda):=\lambda^{\frac{1}{2}} e^{-\lambda}$. We shall prove the theorem for a fixed time interval $[0, T]$ with a constant $C$ independent of $T$. Fix an arbitrary $0<t \leqslant T$. Our starting point is the following identity, valid for $t \in\left[2^{-k} T, 2^{-k+1} T\right):$

$$
\begin{aligned}
\psi(t \lambda) & =\psi\left(2^{-k} T \lambda\right)+\int_{2^{-k} T \lambda}^{t \lambda} \psi^{\prime}(s) d s \\
& =\psi\left(2^{-k} T \lambda\right)+\int_{1}^{2} \mathbf{1}_{\left[2^{-k} s T, 2^{-k+1} T\right)}(t) 2^{-k} s T \lambda \psi^{\prime}\left(2^{-k} s T \lambda\right) \frac{d s}{s} .
\end{aligned}
$$

In order to simplify notations a little bit, thoughout the rest of the proof we take $T=1$. We leave it to the reader to check that the constant $C$ in (6.1) can be chosen independently of $T$.

By the $H^{\infty}$-calculus we have $\psi(-t A)=(-t A)^{\frac{1}{2}} S(t)$. Substituting this in the above identity over $k$, summing over $k=1, \ldots, N$, and writing $\phi(\lambda):=\lambda \psi^{\prime}(\lambda)$, for $t \in\left[2^{-N}, 1\right)$ this gives

$$
\psi(-t A)=\sum_{k=1}^{N} \mathbf{1}_{\left[2^{-k}, 2^{-k+1}\right)}(t) \psi\left(-2^{-k} A\right)+\int_{1}^{2} \sum_{k=1}^{N} \mathbf{1}_{\left[2^{-k} s, 2^{-k+1}\right)}(t) \phi\left(-2^{-k} s A\right) \frac{d s}{s} .
$$

Hence,

$$
\begin{gathered}
\left\|\mathbf{1}_{\left[2^{-N}, 1\right)}(-A)^{\frac{1}{2}} S(\cdot) B\right\|_{\gamma(0,1 ; H, E)}=\left\|\mathbf{1}_{\left[2^{-N}, 1\right)} \psi(-(\cdot) A) B\right\|_{\gamma\left(0,1 ; \frac{d t}{t} ; H, E\right)} \\
\leqslant\left\|\sum_{k=1}^{N} \mathbf{1}_{\left[2^{-k}, 2^{-k+1}\right)} \psi\left(-2^{-k} A\right) B\right\|_{\gamma\left(0,1 ; \frac{d t}{t} ; H, E\right)} \\
\quad+\int_{1}^{2}\left\|\sum_{k=1}^{N} \mathbf{1}_{\left[2^{-k} s, 2^{-k+1}\right)} \phi\left(-2^{-k} s A\right) B\right\|_{\gamma\left(0,1 ; \frac{d t}{t} ; H, E\right)} \frac{d s}{s} .
\end{gathered}
$$

Note that the sequence $\left.\left(\mathbf{1}_{\left[2^{-k}, 2^{-k+1}\right.}\right)\right)_{k=1}^{N}$ is an orthogonal system in $L^{2}\left(0,1 ; \frac{d t}{t}\right)$ with $\left\|\mathbf{1}_{\left[2^{-k}, 2^{-k+1}\right)}\right\|_{2}^{2}=\ln 2$. If $\left(h_{j}\right)_{j \geqslant 1}$ is an orthonormal basis of $H$ and $\left(r_{j k}\right)_{j, k \geqslant 1}$ is a doubly indexed Rademacher sequence on some probability space $(\Omega, \mathscr{F}, \mathbb{P})$, using (2.3) we can estimate

$$
\begin{aligned}
& \left\|\sum_{k=1}^{N} \mathbf{1}_{\left[2^{-k}, 2^{-k+1}\right)} \otimes \psi\left(-2^{-k} A\right) B\right\|_{\gamma\left(0,1 ; \frac{d t}{t} ; H, E\right)}^{2} \\
& =\mathbb{E}\left\|\sum_{j \geqslant 1} \sum_{n=1}^{N} \sum_{k=1}^{N} \gamma_{j n} \int_{0}^{T} \mathbf{1}_{\left[2^{-k}, 2^{-k+1}\right)}(t) \frac{\mathbf{1}_{\left[2^{-n}, 2^{-n+1}\right)}(t)}{\sqrt{\ln 2}} \psi\left(-2^{-k} A\right) B h_{j} \frac{d t}{t}\right\|^{2} \\
& \quad=\ln 2 \cdot \mathbb{E}\left\|\sum_{j \geqslant 1} \sum_{k=1}^{N} \gamma_{j k} \psi\left(-2^{-k} A\right) B h_{j}\right\|^{2} \lesssim \mathbb{E}\left\|\sum_{j \geqslant 1} \sum_{k=1}^{N} r_{j k} \psi\left(-2^{-k} A\right) B h_{j}\right\|^{2}
\end{aligned}
$$


Let $\left(r_{j}^{\prime}\right)_{j \geqslant 1}$ be a Rademacher sequence independent of $\left(r_{j k}\right)_{j, k \geqslant 1}$. Using a randomization argument, we estimate

$$
\begin{aligned}
\mathbb{E}\left\|\sum_{j \geqslant 1} \sum_{k=1}^{N} r_{j k} \psi\left(-2^{-k} A\right) B h_{j}\right\|^{2} & =\mathbb{E}^{\prime} \mathbb{E}\left\|\sum_{j \geqslant 1} r_{j}^{\prime}\left(\sum_{k=1}^{N} r_{j k} \psi\left(-2^{-k} A\right)\right) B h_{j}\right\|^{2} \\
& \leqslant \gamma(\Psi)^{2} \mathbb{E}^{\prime}\left\|\sum_{j \geqslant 1} r_{j}^{\prime} B h_{j}\right\|^{2} \lesssim \gamma(\Psi)^{2}\|B\|_{\gamma(H, E)}^{2} .
\end{aligned}
$$

Here $\gamma(\Psi)$ is the $\gamma$-bound of the family

$$
\Psi=\left\{\sum_{k=1}^{N} r_{j k}(\omega) \psi\left(-2^{-k} A\right): N \geqslant 1, j \geqslant 1, \omega \in \Omega\right\},
$$

which is finite by Lemma 6.1 since $\psi \in H_{\frac{1}{2}}^{\infty}\left(\Sigma_{\sigma}\right)$.

It follows that the function $\sum_{k \geqslant 1} \mathbf{1}_{\left[2^{-k}, 2^{-k+1}\right)} \psi\left(-2^{-k} A\right) B$ defines an almost summing operator from $L^{2}\left(0,1 ; \frac{d t}{t}, H\right)$ to $E$. Since $E$ has finite cotype and therefore does not contain a copy of $c_{0}$, this operator is $\gamma$-radonifying and by (2.1) we have

$$
\left\|\sum_{k \geqslant 1} \mathbf{1}_{\left[2^{-k}, 2^{-k+1}\right)} \psi\left(-2^{-k} A\right) B\right\|_{\gamma\left(0,1 ; \frac{d t}{t} ; H, E\right)} \lesssim \gamma(\Psi)\|B\|_{\gamma(H, E)} .
$$

Likewise, using that for $s \in[1,2)$ the sequence $\left.\left(\mathbf{1}_{\left[2^{-k} s, 2^{-k+1}\right.}\right)\right)_{k=1}^{N}$ is an orthogonal system in $L^{2}\left(0,1 ; \frac{d t}{t}\right)$ with $\left\|\mathbf{1}_{\left[2^{-k} s, 2^{-k+1}\right)}\right\|_{2}^{2}=\ln (2 / s)$,

$$
\begin{aligned}
\int_{1}^{2} \| & \sum_{k=1}^{N} \mathbf{1}_{\left[2^{-k} s, 2^{-k+1}\right)} \phi\left(-2^{-k} s A\right) B \|_{\gamma\left(0,1 ; \frac{d t}{t} ; H, E\right)} \frac{d s}{s} \\
& \lesssim \int_{1}^{2}\left(\ln (2 / s) \cdot \mathbb{E}\left\|\sum_{k=1}^{N} \sum_{j \geqslant 1} r_{j k} \phi\left(-2^{-k} s A\right) B h_{j}\right\|^{2}\right)^{\frac{1}{2}} \frac{d s}{s} \\
& \leqslant \gamma(\Phi) \int_{1}^{2} \ln (2 / s) \cdot\left(\mathbb{E}^{\prime}\left\|\sum_{j \geqslant 1} r_{j}^{\prime} B h_{j}\right\|^{2}\right)^{\frac{1}{2}} \frac{d s}{s} \lesssim \gamma(\Phi)\|B\|_{\gamma(H, E)} .
\end{aligned}
$$

Here $\gamma(\Phi)$ is the $\gamma$-bound of the family

$$
\Phi=\left\{\sum_{k=1}^{N} r_{j k}(\omega) \phi\left(-2^{-k} s A\right): N \geqslant 1, j \geqslant 1, s \in[1,2], \omega \in \Omega\right\},
$$

which is finite since $\phi \in H_{\frac{1}{2}}^{\infty}\left(\Sigma_{\sigma}\right)$. Letting $N \rightarrow \infty$ as before, with monotone convergence it follows that

$$
\int_{1}^{2}\left\|\sum_{k \geqslant 1} \phi\left(-2^{-k} s A\right) B \mathbf{1}_{\left[2^{-k} s, 2^{-k+1}\right)}(\cdot)\right\|_{\left.\gamma\left(0,1 ; \frac{d t}{t} ; H\right) E\right)} \frac{d s}{s} \lesssim \gamma(\Phi)^{2}\|B\|_{\gamma(H, E)}^{2} .
$$

As $N \rightarrow \infty$ we also obtain that $(-A)^{\frac{1}{2}} S(\cdot) B \in \gamma(0,1 ; H, E)$ and

$$
\left\|(-A)^{\frac{1}{2}} S(\cdot) B\right\|_{\gamma(0,1 ; H, E)}=\lim _{N \rightarrow \infty}\left\|(-A)^{\frac{1}{2}} S(\cdot) B \mathbf{1}_{\left[2^{-N}, 1\right)}\right\|_{\gamma(0,1 ; H, E)} .
$$

Putting things together we obtain that

$$
\left\|(-A)^{\frac{1}{2}} S(\cdot) B\right\|_{\gamma(0,1 ; H, E)} \leqslant C\|B\|_{\gamma(H, E)}
$$

with a constant $C$ independent of $B$. Therefore, for all $t \in[0,1]$ the function $(-A)^{\frac{1}{2}} S(t-\cdot) B$ is $H$-stochastically integrable, and (6.1) follows from

$$
\begin{aligned}
\mathbb{E}\left\|(-A)^{\frac{1}{2}} U(t)\right\|^{2} & =\left\|(-A)^{\frac{1}{2}} S(t-\cdot) B\right\|_{\gamma(0, t ; H, E)}^{2} \\
& \leqslant\left\|(-A)^{\frac{1}{2}} S(\cdot) B\right\|_{\gamma(0,1 ; H, E)}^{2} \leqslant C\|B\|_{\gamma(H, E)} .
\end{aligned}
$$


This proves (6.1). By Fubini's theorem, (6.1) implies that

$$
\mathbb{E} \int_{0}^{T}\left\|(-A)^{\frac{1}{2}} U(t)\right\|^{2} d t \leqslant T C\|B\|_{\gamma(H, E)}^{2}
$$

Hence the paths of $(-A)^{\frac{1}{2}} U$ belong to $L^{2}(0, T ; E)$ almost surely. Finally the continuity in all moments follows from [23, Theorem 6.5].

Remark 6.3. The theorem remains true if only $\nu-A$ admits a $\gamma$-bounded $H^{\infty}$ calculus for some $\nu>0$. To see this we apply the theorem with $-A$ replaced by $\nu-A$ to obtain maximal regularity of the solution of the problem

$$
\left\{\begin{aligned}
d U(t) & =(A-\nu) U(t) d t+B d W_{H}(t), \quad t \in[0, T], \\
U(0) & =0
\end{aligned}\right.
$$

We obtain that $(\nu-A)^{\frac{1}{2}} S_{\nu}(\cdot) B \in \gamma(0, T ; H, E)$, where $S_{\nu}(t)=e^{-\nu t} S(t)$. By a standard comparison argument this implies that also $(\nu-A)^{\frac{1}{2}} S(\cdot) B \in \gamma(0, T ; H, E)$, with similar estimates.

Remark 6.4. In the special case where $H=\mathbb{R}$, Lemma 6.1 is not needed and Theorem 6.2 remains valid under the weaker assumption that $-A$ admits a bounded $H^{\infty}$-calculus.

As is well known, the deterministic Cauchy problem $y^{\prime}=A y+f$, with $-A$ sectorial of angle $0<\omega(-A)<\frac{\pi}{2}$, has maximal $L^{p}$-regularity if and only if the set $\{t R(i t, A): t \in \mathbb{R} \backslash\{0\}\}$ is $R$-bounded (see [31]). The following result shows that in the stochastic setting, the strictly stronger assumption that $-A$ admits a bounded $H^{\infty}$-calculus is necessary for maximal regularity and actually characterizes it in the case $H=\mathbb{R}$ (which corresponds to rank one Brownian motions). In particular this shows that in $L^{p}$-spaces there are examples of analytic generators which have maximal regularity for the deterministic Cauchy problem but not always for the stochastic one.

We use the notation $E^{\odot}$ for the closed subspace of all $x^{*} \in E^{*}$ such that $\lim _{t \downarrow 0}\left\|S^{*}(t) x^{*}-x^{*}\right\|=0$. As is well known we have $E^{\odot}=\overline{\mathscr{D}\left(A^{*}\right)}$. The part of $A^{*}$ in $E^{\odot}$ is denoted by $A^{\odot}$; it is the generator of the restriction of $S^{*}$ to $E^{\odot}$.

Theorem 6.5. Let both $E$ and $E^{*}$ have finite cotype, and let $-A$ be a sectorial operator in $E$ of angle $0<\omega(-A)<\frac{\pi}{2}$. Then $-A$ admits a bounded $H^{\infty}$-calculus if and only if

$$
d U=A U d t+x d W(t), \quad t \geqslant 0
$$

and

$$
d \tilde{U}=A^{\odot} U^{\odot} d t+x^{\odot} d W(t), \quad t \geqslant 0,
$$

have maximal regularity in the sense of Theorem 6.2 for all $x \in E$ and $x^{\odot} \in E^{\odot}$, respectively.

Proof. The 'only if' part is contained in the previous theorem and the remark following it, since $-A$ admits a bounded $H^{\infty}$-calculus if and only if $-A^{\odot}$ admits a bounded $H^{\infty}$-calculus $[11,18]$.

For the 'if' part, for all $t \in[0, T]$ we have

$$
\left\|(-A)^{\frac{1}{2}} S(\cdot) x\right\|_{\gamma(0, t ; E)}^{2}=\left\|(-A)^{\frac{1}{2}} S(t-\cdot) x\right\|_{\gamma(0, t ; E)}^{2}=\mathbb{E}\left\|(-A)^{\frac{1}{2}} U(t)\right\|^{2} \leqslant C\|x\|^{2}
$$

with a constant $C$ independent of $t, T$, and $x$. Likewise,

$$
\left\|\left(-A^{\odot}\right)^{\frac{1}{2}} S^{\odot}(\cdot) x^{\odot}\right\|_{\gamma(0, t ; E \odot)}^{2} \leqslant C\left\|x^{\odot}\right\|_{\gamma(H, E \odot)}^{2} .
$$

By [16], these two estimates imply that $-A$ admits a bounded $H^{\infty}$-calculus. 
Remark 6.6. If $0 \in \varrho(A)$ it suffices to assume the existence of solutions on bounded intervals $[0, T]$ and the constant $C$ in (6.1) is allowed to depend on $T$.

\section{An EXAMPle}

Let $\mathscr{O}$ be a bounded open domain in $\mathbb{R}^{d}$ with $C^{2}$ boundary. Consider the problem

$$
\left\{\begin{array}{rrr}
d u(t, x)=L u(t, x) d t+\sum_{k=1}^{\infty} g_{k}(x) d w_{k}(t), & & x \in \mathscr{O}, t \in[0, T], \\
u(0, x)=0, & x \in \mathscr{O}, \\
u(t, x)=0, & x \in \partial \mathscr{O}, t \in[0, T],
\end{array}\right.
$$

where $L$ is a second order uniformly elliptic operator of the form

$$
L f(x)=\sum_{i, j=1}^{d} a_{i j}(x) \frac{\partial^{2} f}{\partial x_{i} \partial x_{j}}(x)+\sum_{i=1}^{d} b_{i}(x) \frac{\partial f}{\partial x_{i}}(x)+c(x) f(x), \quad x \in \mathscr{O},
$$

with coefficients $a_{i j}=a_{j i} \in C^{\epsilon}(\overline{\mathscr{O}})$ for some $\epsilon>0$ and $b_{i}, c \in L^{\infty}(\mathscr{O})$ with $c \leqslant 0$. We assume that the sequence $g=\left(g_{k}\right)_{k \geqslant 1}$ belongs to $L^{p}\left(\mathscr{O} ; l^{2}\right)$ for some fixed $1<p<\infty$, and that $w=\left(w_{k}\right)_{k \geqslant 1}$ is a sequence of independent standard Brownian motions. A related, time-dependent version of this equation on the full space $\mathbb{R}^{d}$ has been considered by Krylov [17, Chapter 5.4].

Here we will show that (7.1) has a unique solution in $L^{p}(\mathscr{O})$, with paths belonging to $C^{\beta}\left([0, T] ; L^{p}(\mathscr{O})\right) \cap L^{2}\left(0, T ; H_{0}^{1, p}(\mathscr{O})\right)$ for $0 \leqslant \beta<\frac{1}{2}$.

Let $1<p<\infty$ and take $E=L^{p}(\mathscr{O})$. In $E$ we consider the realization $A$ of $L$ with Dirichlet boundary conditions, i.e., $\mathscr{D}(A)=H^{2, p}(\mathscr{O}) \cap H_{0}^{1, p}(\mathscr{O})$. Let $\left(e_{k}\right)_{k \geqslant 1}$ denote the standard unit basis of $l^{2}$, and define $B \in \mathscr{L}\left(l^{2}, L^{p}(\mathscr{O})\right)$ by $B h:=\sum_{k \geqslant 1}\left[h, e_{k}\right]_{l^{2}} g_{k}$ for $h \in l^{2}$. We can rewrite (7.1) as a linear stochastic Cauchy problem of the form

$$
\left\{\begin{aligned}
d U(t) & =A U(t) d t+B d W_{l^{2}}(t), \quad t \in[0, T], \\
U(0) & =0
\end{aligned}\right.
$$

with $H_{l^{2}}$ an $l^{2}$-cylindrical Brownian motion. The operator $B$ is $\gamma$-radonifying since by the Fubini theorem and the Kahane-Khinchine inequalities,

$$
\begin{aligned}
\mathbb{E}\left\|\sum_{k \geqslant 1} \gamma_{k} B e_{k}\right\|_{L^{p}}^{2} & \lesssim p \\
& =\left.\int_{\mathscr{O}} \mathbb{E}\left|\sum_{k \geqslant 1} \gamma_{k} B e_{k}\left\|_{L^{p}}^{p}=\mathbb{E}\right\| \sum_{k} g_{k}(x)\right|_{k}\right|_{k} g_{k} \|_{L^{p}}^{p}
\end{aligned}
$$

which is finite by the assumption on $g$. It was shown in [10] that $\nu-A$ admits a bounded $H^{\infty}$-calculus for $\nu>0$ sufficiently large. This calculus is $\gamma$-bounded since $L^{p}(\mathscr{O})$ has finite. It follows that the assumptions of Theorems 4.1 and 6.2 (with $A$ replaced $A-\nu$ ) by are satisfied. Since $A$ is invertible we have $\mathscr{D}\left((-A)^{\frac{1}{2}}\right)=$ $\mathscr{D}\left((\nu-A)^{\frac{1}{2}}\right)=H_{0}^{1, p}(\mathscr{O})$ with equivalent norms (see [10, 27, 28]). By Theorem 6.2 and the remark following it we obtain a unique solution $U$ of (7.2) with paths belonging to $C^{\beta}\left([0, T] ; L^{p}(\mathscr{O})\right) \cap L^{2}\left(0, T ; H_{0}^{1, p}(\mathscr{O})\right)$ for $0 \leqslant \beta<\frac{1}{2}$.

Acknowledgment - Part of this work was done during a half-year stay of the first named author at the Delft Institute of Applied Mathematics at the Technical University of Delft. The authors thank Philippe Clément, Guido Sweers, and Mark Veraar for useful comments. 


\section{REFERENCES}

[1] S. Agmon, "Lectures on Elliptic Boundary Value Problems", Van Nostrand Mathematical Studies, No. 2, Princeton, 1965.

[2] V.I. Bogachev, "Gaussian Measures", Math. Surveys and Monographs, Vol. 62, Amer. Math. Soc., Providence, RI, 1998.

[3] Z. BrZeŹniaK, On stochastic convolutions in Banach spaces and applications, Stoch. Stoch. Reports 61 (1997), 245-295.

[4] Z. Brzeźniak and J.M.A.M. van Neerven, Stochastic convolution in separable Banach spaces and the stochastic linear Cauchy problem, Studia Math. 143 (2000), 43-74.

[5] S. Cerrai, "Second Order PDE's in Finite and Infinite Dimension. A Probabilistic Approach", Springer Lect. Notes Math. 1762, Springer-Verlag, Berlin, 2001

[6] P. Clément, B. de Pagter, F. A. Sukochev and H. Witvliet, Schauder decompositions and multiplier theorems, Studia Math. 138 (2000), 135-163.

[7] M. Cowling, I. Doust, A. McIntosh, And A. Yagi, Banach space operators with a bounded $H^{\infty}$ functional calculus, J. Austral. Math. Soc. Ser. A 60 (1996), 51-89.

[8] G. Da Prato and J. Zabczyk, Nonexplosion, boundedness, and ergodicity for stochastic semilinear equations, J. Differential Equations 98 (1992), 181-195.

[9] G. Da Prato and J. Zabczyk, "Stochastic Equations in Infinite Dimensions", Encyclopedia of Mathematics and its Applications, Cambridge University Press, Cambridge, 1992.

[10] R. Denk, G. Dore, M. Hieber, J. Prüss, And A. Venni, New thoughts on old results of R. T. Seeley, Math. Ann. 328 (2004), 545-583.

[11] R. Denk, M. Hieber, And J. Prüss, " $R$-Boundedness, Fourier Multipliers and Problems of Elliptic and Parabolic Type", Mem. Amer. Math. Soc. 166, 2003.

[12] J. Dettweiler and J.M.A.M. van Neerven, Continuity versus nonexistence for a class of linear stochastic Cauchy problems driven by a Brownian motion, to appear in Czech. Math. J.

[13] J. Diestel, H. Jarchow And A. Tonge, "Absolutely Summing Operators", Cambridge Studies in Adv. Math., Vol. 34, Cambridge, 1995.

[14] K.-J. Engel and R. Nagel, "One-Parameter Semigroups for Linear Evolution Equations", Graduate Texts Math., Vol. 194, Springer-Verlag, 2000.

[15] N.J. Kalton And L. Weis, The $H^{\infty}$-calculus and sums of closed operators, Math. Ann. 321 (2001), 319-345.

[16] N.J. Kalton and L. Weis, The $H^{\infty}$-functional calculus and square function estimates, preprint.

[17] N.V. Krylov, An analytic approach to SPDEs, in: "Stochastic Partial Differential Equations: Six Perspectives", 185-242, Math. Surveys Monogr., Vol. 64, Amer. Math. Soc., Providence, R.I., 1999.

[18] P.Ch. Kunstmann and L. Weis, Maximal $L_{p}$-Regularity for Parabolic Equations, Fourier Multiplier Theorems and $H^{\infty}$-Functional Calculus, in: "Levico Notes on Evolution Equations and Semigroups", Springer Lect. Notes Math. 1855, Springer-Verlag, 2004.

[19] C. Le Merdy, The similarity problem for bounded analytic semigroups on Hilbert spaces, Semigroup Forum 56 (1998), 205-224.

[20] M. Ledoux and M. Talagrand, "Probability in Banach Spaces", Ergebnisse d. Math. u. ihre Grenzgebiete, Vol. 23, Springer-Verlag, 1991.

[21] A. Lunardi, "Analytic Semigroups and Optimal Regularity in Parabolic Problems", Progress in Nonlinear Differential Equations and their Applications, Vol. 16, Birkhäuser Verlag, Basel, 1995.

[22] A. LunARd, "Interpolation Theory", Appunti, Scuola Normale Superiore Pisa, 1999.

[23] J.M.A.M. NeERVEn AND L. WeIs, Stochastic integration of functions with values in a Banach space, Studia Math. 166 (2005), 131-170.

[24] J.M.A.M. NEERVEN AND L. WEIS, Stochastic integration of operator-valued functions with respect to Banach space-valued Brownian motion, submitted for publication.

[25] A.L. Neidhardt "Stochastic Integrals in 2-Uniformly Smooth Banach Spaces", Ph.D. Thesis, University of Wisconsin, 1978.

[26] G. PISIER, Some results on Banach spaces without local unconditional structure, Compositio Math. 37 (1978), 3-19.

[27] J. PRÜss AND H. SoHR, Imaginary powers of elliptic second order differential operators in $L^{p}$-spaces, Hiroshima Math. J. 23 (1993), 161-192.

[28] R. Seeley, Interpolation in $L^{p}$ with boundary conditions, Studia Math. 44 (1972), 47-60.

[29] N.N. Vakhania, V.I. Tarieladze and S.A. Chobanyan, "Probability Distributions on Banach Spaces", D. Reidel Publishing Company, Dordrecht-Boston-Lancaster-Tokyo, 1987.

[30] M.C. Veraar and J. Zimmerschied, Non-autonomous stochastic Cauchy problems in Banach spaces, submitted. 
[31] L. WeIs, Operator-valued Fourier multiplier theorems and maximal $L_{p}$-regularity, Math. Ann. 319 (2001), 735-758.

Mathematisches Institut I, Technische Universität Karlsruhe, D-76128 KarlsRuhe, Germany

E-mail address: Johanna.Dettweiler@math.uni-karlsruhe.de

Delft Institute of Applied Mathematics, Technical University of Delft, P.O. Box 5031, 2600 GA Delft, The Netherlands

E-mail address: J.vanNeerven@math.tudelft.nl

Mathematisches Institut I, Technische Universität KarlsRuhe, D-76128 KarlsRuhe, Germany

E-mail address: Lutz.Weis@math.uni-karlsruhe.de 